Review Article

\title{
IMPORTANCE OF HAIR GROWTH IN HISUTISM: DIAGNOSIS AND TREATMENT
}

\author{
AANCHHAL, RAHUL MEHRA, AMIT BARWAL, SURYA PARKASH GAUTAM
}

Department of pharmacology, CT Group of Institutions, Jalandhar, India 144020

Email: aanchalpalta98@gmail.com

Received: 04 Aug 2021, Revised and Accepted: 10 Oct 2021

\begin{abstract}
The objective of the review is to explain the pathogenesis, causes and various treatment involved in hirsutism. This article discusses the disease's pathogenesis, causes and diagnosis. This review looks at the main significant type of hairs and clinical studies on the role of several lifestyle therapies in hirsutism development. This review examines the numerous methods that causes hirsutism in order to discover new medicaments. In addition, it covers the various type of hirsutism therapy. Hirsutism, is reported to have the strongest impact on patients' health-related quality of life, following in descending order by body mass index, irregular menses, and infertility. To assess the types of hairs and pathogenesis, sign and symptoms, as well as causes of hirsutism. Moreover, we studied the management of hirsutism and how to treat this. At least six to nine months of therapy are required to produce improvement in hirsutism. We suggest testing for elevated androgen levels in women with moderate or severe hirsutism or hirsutism of any degree when it is sudden in onset, rapidly progressive, or associated with other abnormalities such as menstrual dysfunction, obesity, or macroclitoris. For women with patient-important hirsutism despite cosmetic measures, we suggest either pharmacological therapy or direct hair removal methods. For pharmacological therapy, we suggest oral contraceptives for the majority of women, adding an Antiandrogens after 6 mo if the response is suboptimal. We recommend against androgen antagonist monotherapy unless adequate contraception is used. We suggest against using insulin-lowering drugs. For women who choose hair removal therapy, we suggest laser/photo epilation.
\end{abstract}

Keywords: Hirsutism, Hypertrichosis, Hair growth, Diagnosis, Treatment

(c) 2021 The Authors. Published by Innovare Academic Sciences Pvt Ltd. This is an open access article under the CC BY license (https://creativecommons.org/licenses/by/4.0/) DOI: https://dx.doi.org/10.22159/ijcpr.2021v13i6.1914 Journal homepage: https://innovareacademics.in/journals/index.php/ijcpr

\section{INTRODUCTION}

The medical word for excessive terminal (coarse) hair in androgensensitive regions of the female body is hirsutism (upper lip, chin, chest, back, abdomen, arms, and thighs). It should be distinguished from virilisation, which refers to the coexistence of hirsutism and a wide range of androgen excess symptoms, such as ambiguous external genitalia, increased muscle mass, acne, balding, deepening of the voice, breast atrophy, amenorrhea/oligomenorrhoea, and increased libido, which varies with age [1]. Depending on the patient's age, all of these signs and symptoms may have different clinical presentations. Hypertrichosis is hair growth that is abnormal for an individual's age, sex, or race, or for a specific area of the body, and should be distinguished from hirsutism [1]. This condition is known as 'hypertrichosis,' which refers to a rise in hair ('hyper') and trichosis ('trichosis'). Only hirsutism will be discussed in this pamphlet.

Androgenic factors, non-androgenic factors, and idiopathic hirsutism are three types of hirsutism causes. Non-androgenic reasons are uncommon, while androgenic causes account for more than $80 \%$ of cases, including polycystic ovarian syndrome (PCOS), which affects around $70 \%$ to $80 \%$ of hirsute women. Hirsutism is distinguished from hypertrichosis, which is characterised by excessive hair growth in a asexual agamic, generalised pattern and is not caused by androgens. Hypertrichosis usually manifests as vellus growth (no hair on the body) that is either widespread or localised. Drugs, genetic factors, metabolic or other non-endocrine diseases can all induces hypertrichosis, but it is not caused by too much testosterone, as previously stated. Regardless, androgens have the potential to exacerbate the issue. Hypertrichosis has been linked to a broad range of medications. Acetazolamide, corticotrophin, cyclosporine, diazoxide, glucocorticoids, Metoclopramide, methyldopa, mercure poisoning, minoxidil, penicillamine, phenothiazines, phenytoin, reserpine, streptomycin, valproic acid, and heavy metals are examples of these medicines [2, 3].

Hypertrichosis may typically be cured within weeks or months of stopping the medicines in these situations. Patients with systemic diseases such as porphyria, constipation, starvation, juvenile dermatomyositis, TB, hypothyroidism, and even Paranephric syndrome might develop hypertrichosis. A modified Ferriman and Gallwey scale is a widely used method for grading hair growth (fig. 1). Hirsutism has been defined as a score of eight or higher [4, 5].

\section{Development and types of hair}

There are roughly 50 million hair follicles on the body, with 100000 to 150000 on the scalp and the rest of the face and other parts of the body. The soles of the feet, palms of the hands, and lips are the only parts of the body without hair follicles. After the age of 40 , the number of hair follicles begins to decline, since new hair follicles produces seldom. During the development of hair structures, the primitive mesoderm forms the hair germ, with an associated down growth sending ectoderm cells to the newly created hair peg mesoderm during the formation of hair peg.

The hair peg develops a solid core of epithelial cells that encloses the dermal papilla, while the hair germ from the mesoderm produces the fibrous sheath of the follicle and the dermal papilla. The ectodermal and mesodermal components stay in close touch throughout the life of the hair follicle, indicating a long-term relationship. There are three sorts of hair in terms of structure.

1. Lanugo is silky hair that covers the skin of the foetus and sheds between the first and fourth month after delivery. Peach fuzz hairs are similar to lanugo hairs in that they are soft and fine, but they are bigger.

2. Vellus hairs are non-pigmented and measure less than $2 \mathrm{~mm}$ in length, and they cover the body's ostensibly hairless regions. Baby hairs have a diameter of less than $0.03 \mathrm{~mm}$, which is less than the diameter of the investing root sheath. The terminal hairs are thicker, more pigmented, and have a coarse feel. The eyebrows, eyelashes, scalp hair, pubic and axillary hair in both sexes, as well as most of the body and facial hair of males, are all made up of this hair.

3. Terminal hairs are frequently referred to as "medullated." The innermost region of terminal hairs is called the "medulla" of the hair follicle. The hairs of the lanugo and baby hairs are not medullated [7]. 


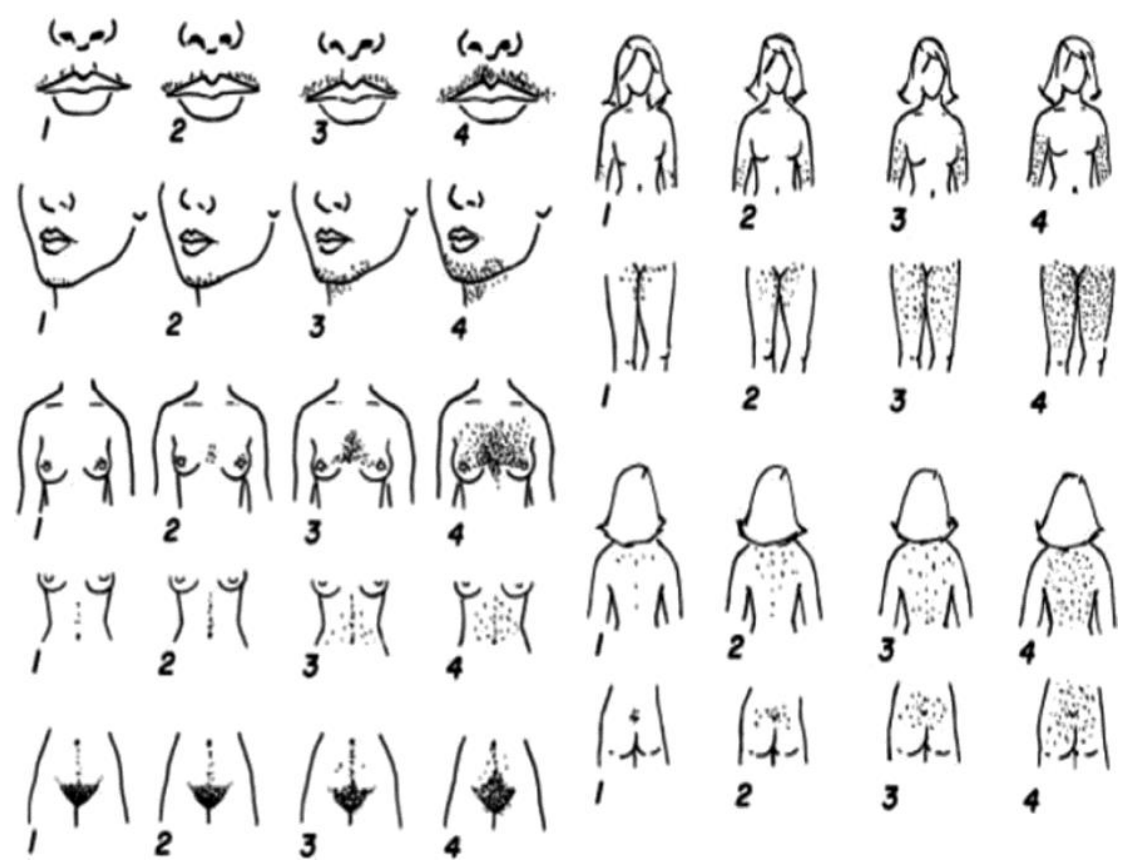

Fig. 1: The modified ferriman-gallwey (F-G) hirsutism grading system is shown in Each of the nine body regions is scored from zero (no terminal hairs) to four (abundant terminal hair development), and the overall score is calculated by adding the numbers in each area [6].

1997, Ricardo Azziz

\section{Growth phases of hair}

Hair development occurs in three stages. An active growth phase is followed by an involutional stage (transitional), during which the hair stops growing and the hair bud shrinks; and finally, the telogen phase, during which the hair rests and is lost while new hairs replace it. Catagen and telogen stages span 2 to $3 \mathrm{w}$ and 3 to $4 \mathrm{mo}$, respectively, in scalp and body hair.

The resting phase-transitional ratio (the ratio of anagen hairs to telogen hairs) is used to evaluate hair growth activity in specific regions of the skin, with a greater ratio suggesting more active hair growth [7].

Local growth factors and circulating androgens boost peripheral 5reductase activity. This enzyme is responsible for converting testosterone to Dihydrotestosterone (DHT). DHT increases the following in body hair:

1. Increased sebum production

2. The transition of transitional stage to terminal hairs in the hair follicle; and

3. The growth phase is extended, resulting in longer, thicker hairs.

\section{Pathogenesis of hirsutism}

Hirsutism can be caused by an increase in androgen levels or by the hair follicles being oversensitive to androgen. Sex hormone-binding globulin (SHBG), cortisol-binding globulin (CBG), and albumin bind large amounts of circulating androgens. The major bioactive component of plasma testosterone is free testosterone. SHBG levels can drop in the body due to a variety of factors, including obesity, Hyperinsulinemia, and the use of androgens, synthetic progestins, glucocorticoids, and growth hormones. The drop in SHBG levels in the body causes an increase in free testosterone, which can lead to hirsutism. However, the degree of hirsutism does not seem to be related to androgen levels. This is due to the fact that the sensitivity of hair follicles to androgen differs from person to person. 5 alphareductase type 1 convertes the testosterone to the highly active dihydrotestosterone [8].

According to a recent update on hirsutism, family clustering of PCOS, hyperandrogenism, and hirsutism suggests a genetic cause. In certain families, environmental variables may also be the triggering agents. The idea that the intrauterine environment influences the hyperandrogenic phenotype in adult life is supported by recent experimental evidence [8]. Insulin and LH both increase androgen synthesis in ovarian theca cells. As a result, the ovaries release more testosterone and Androstenedione than normal [9]. Insulin resistance that is not caused by obesity has also been identified as a pathognomonic feature of PCOS [10]. Menstrual abnormalities, hyperandrogenism, hyperinsulinemia, and long-term metabolic disturbances such as diabetes mellitus, cardiovascular disease, and dyslipidemia are all symptoms of PCOS [11]. Insulin and body fat both have a role in controlling lipid levels [9]. While both DHT and testosterone transform short, soft baby hair into coarse, DHT is considerably more efficient than testosterone. Conversion is irreversible and hence only hairs are converted to end hair in androgen-sensitive areas [9].

\section{Causes}

One symptom of oligomenorrhea is hirsutism. Excess androgen production by the ovaries and adrenal glands, as well as exogenous sources of androgens such as medicines, can cause hirsutism.

- Idiopathic hirsutism is frequently inherited. The condition begins shortly after puberty and progresses slowly. Menstruation is normal, and testosterone levels are normal [12]

- PCOS, androgen-secreting ovarian tumours, and menopause are all ovarian causes. Oligomenorrhea and polycystic ovaries are the hallmarks of PCOS [12].

- Tumors, CAH, and Cushing's syndrome are all causes of adrenal insufficiency.

- The following medications cause drug-induced hirsutism:

- Metoclopramide

- Phenothiazines are a kind of phenothiazines.

- Progestins are hormones that are produced by the female reproductive system.

- Reserpine is a kind of reserpine that

- Testicles is produced by the testosterone hormone. 
Severe insulin resistance, anorexia nervosa, hyperprolactinemia, acromegaly, hypothyroidism, and porphyria are among the other reasons. Virilization is frequently associated with androgensecreting tumours of the ovary or adrenal gland. Virilization is caused by arrhenoblastoma, sertoli leydig cell tumour, theca cell tumour, and some instances of granulosa cell tumour.

\section{Virilization signs include}

- Acne

\section{- Seborrhea}

- Macroclitoris is a condition in which a person has a lot of

- An increase in the volume of one's voice

- Boosted libido

- Muscle mass increase

- Menses that are irregular or non-existent

- Balding and temporary hair loss.

Virilization, fast advancement of hirsutism, and the cessation of menses are all signs of androgen-secreting tumours of the ovary or adrenal. Adrenal tumours that secrete androgen are less common [13].

Although hirsutism is the most prevalent symptom of hyperandrogenism, virilization symptoms imply elevated blood androgen levels. Cushing's syndrome should be explored in the differential diagnosis, despite its rarity. It might be caused by increased pituitary adrenocorticotropic hormone production, adrenal carcinoma/adenoma, or adrenocorticotropic hormone secretion. The most prevalent cause of profound hirsutism is micronodular hyperplasia [12].

\section{Diagnosis}

Although most women seek medical help because of the discomfort of hirsutism, clinical evaluation is done to check whether there is an endocrinologic disease that warrants it. Because there is no definitive clinical difference between physiologic and pathologic hirsutism, family history and physical examination are especially essential in evaluating excessive hair growth in women. Idiopathic hirsutism affects the great majority of women, and diagnosis is determined by exclusion [14].

Once hirsutism has been diagnosed, it's a good idea to look for additional androgen excess symptoms, including intractable acne, female-pattern alopecia, and seborrhea. Clitoromegaly, a deeper voice, malepattern alopecia and loss of feminine body shape are all warning indicators of virilization [14].

The Endocrine Society recently published a clinical practice guideline that advises against testing for increased androgen levels in women with mild hirsutism (FG score 8-15), citing the fact that hyperandrogenemia is undetectable in about half of these cases using conventional laboratory tests and the low likelihood of identifying a medical disorder that would change management or outcome. Women with moderate to severe hirsutism, as well as women with any degree of hirsutism, should have their androgen levels tested if their hirsutism is sudden in onset, rapidly progressive, or associated with any of the following: menstrual irregularity, central obesity, acanthosis nigricans, or clitoromegaly $[15,16]$.

\section{Medical history}

Menstrual history, beginning and progression of hairiness, weight gain, current or past therapies, and birth history of hyperandrogenism should all be included in a complete medical history. There is a growing body of data that women with PCOS, hyperandrogenism and metabolic changes have a family history [18]. A cycle duration of more than $35 \mathrm{~d}$ in adult women may indicate oligomenorrhea [19].

\section{Physical examination}

A thorough medical history and physical examination are frequently sufficient to rule out pathologic causes of hirsutism. The modified
Ferriman-Gallwey score is a qualitative instrument for assessing and measuring female hair growth in nine androgen-dependent regions. This grading method assesses nine body areas (upper lip, chin, chest, upper back, lower back, upper abdomen, lower abdomen, arm, and thigh), with scores ranging from zero (no excessive terminal hair growth evident) to four (extensive hair growth visible) for each. A maximum score of 36 is conceivable, although a score of 8 generally suggests hirsutism, according to Ferriman's 95th percentile data [14].

Because of the subjective nature of the assessments and the difficulties of grading women who have cosmetically lost their hair, this scoring system has limitations [20]. A dermatologic examination is required because elevated androgen levels can produce pilosebaceous.

The patient's height and weight should be noted, as well as their BMI (body mass index). It's also a good idea to check your blood pressure [16]. Acanthosis nigricans (indicative of insulin resistance), acne vulgaris (especially if there are premenstrual cysts and nodules on the skin), seborrhea, and androgenetic (patterned) alopecia are some of the skin findings [16].

Signs and symptoms of virilization, such as deeper voice, clitoromegaly, breast atrophy, and increased muscular mass, should be evaluated during the clinical examination. To rule out an ovarian or adrenal tumour mass, palpate the abdomen and pelvis [16]. It is critical to assess a visual field defect.

Patients should also be asked about changes in their quality of life and screened for depressive symptoms (such as sleeping difficulties, loss of energy, and drive). Disgust, changes in sexual activity, life behavior, and life events, as well as symptoms of body dysmorphic disorder, should all be considered. Additional counselling or psychotherapy should be considered if required [21].

Women with PCOS have recently been linked to an increased risk of mood and anxiety problems. Independent of BMI, women with PCOS have greater depression ratings and a higher risk of depression. Despite the fact that clinical characteristics of hyperandrogenism have an impact on unfitness quality of life, the link between hirsutism, acne, body image, and depression is still unknown. Similarly, research on the relationship between factors such biochemical hyperandrogenism or infertility and depression is scarce. Symptoms of generalised anxiety disorder are also a danger for women with PCOS. The risk of additional anxiety disorders, such as social phobia, obsessive-compulsive disorders, and panic disorder, is not well understood. Some of these illnesses coexist in a number of individuals, adding to the health burden. These findings highlight the need of screening all women with PCOS for mood and anxiety problems, as well as providing sufficient treatment to those who are identified [22, 23].

\section{Evaluation in the lab}

If a drug is the reason, a simple drug withdrawal should be beneficial. In all other situations, serum indicators should be evaluated in the lab to determine the precise cause. The different serum indicators are as follows:

\section{Testosterone}

According to clinical practice recommendations published by the Endocrine Society in 2008[15]. They advise avoiding testing for increased androgen levels in women with isolated mild hirsutism since the chances of discovering a medical condition that would affect therapy or outcome are slim. However, in women with moderate or severe hirsutism, they recommend testing for increased androgen levels.

- Hirsutism of any degree when it appears suddenly progresses quickly, or is accompanied for any of the following: -

- Infertility or menstrual irregularities

- Obesity in the centre.

- Acanthosis nigricans is a kind of acanthosis.

- Fast advancement 


\section{- Clitoromegaly}

The most accurate way to determine androgen levels is to use a specialised laboratory [24]. In women, the typical maximum range for total plasma testosterone is between 70 and $90 \mathrm{ng} / \mathrm{dl}$ (2.43 and 3.12 $\mathrm{nmol} / \mathrm{l})$. This is because tests differ in a systematic way32, and many laboratories offer abnormally large normal ranges since the general population includes women with undiagnosed androgen excess $[25,26]$. Serum testosterone levels may be normal to slightly elevate in benign pathologies such as PCOS and CAH, but they will be significantly elevated ( $>200 \mathrm{ng} / \mathrm{ml}$ ) in malignant tumours of the adrenal or ovary [27].

\section{Dehydroepiandrosterone sulphate (DHEAS)}

Routine androgen testing is ineffective [29-31]. Dehydroepiandrosterone sulphate levels are elevated in around $15 \%$ of women with normal total and free testosterone levels. Apart from being linked to acne, a modestly increased level in a woman with a normal free testosterone level is unlikely to be clinically relevant [32-34]. DHEAS elevation ( $>700 \mathrm{~g} / \mathrm{dl}$ ) is usually indicative of an adrenal origin, whether benign or malignant [28].

\section{7-hydroxyprogesterone}

This blood marker is only seen in people with congenital adrenal hyperplasia. In the early follicular phase of the menstrual cycle, the measurement should be taken between 0700 and $0900 \mathrm{~h}$. The illness is ruled out if the level is less than $200 \mathrm{ng} / \mathrm{dl}$. An ACTH stimulation test is required for mildly elevated values between 300 and 1,000 $\mathrm{ng} / \mathrm{dl}$. A 250-gram dose of cosyntropin (synthetic ACTH) is given intravenously, and 17-hydroxyprogesterone levels are tested before and one hour after the administration. A positive test is defined as a post-stimulation result of $>1,000 \mathrm{ng} / \mathrm{dl}[29]$.

d) In women with indications and symptoms of Cushing's syndrome, urine-free cortisol should be tested after $24 \mathrm{~h}$.

e) A ratio of LH/FSH greater than 3 indicates PCOS [30].

f) In hyperprolactinemia, prolactin levels would be elevated due to hypothalamic illness or a pituitary tumour.

g) Hypophyseal hypothyroidism (serum TSH) [31]. In hirsutism, it can serve as a cofactor, producing an increase in TSH.

\section{Imaging}

Diagnostic imaging to determine the site of the tumour is useful in directing therapy when an adrenal or ovarian neoplasm is suspected [32]. If a patient has amenorrhea and/or indications of virilization, a pelvic exam is required [20]. If there is a suspicion of pituitary dysfunction, central nervous system imaging may be taken.

\section{Treatment}

The degree of excess hair growth displayed by the patient, as well as the pathophysiology of the condition, should be considered while treating hirsutism. The patient's expectations of therapy should be addressed. She should be told that total eradication with medication treatment is improbable, but it may be reduced by making the treatment less severe and requiring longer intervals between cosmetic treatments (shaving, plucking, waxing). The majority of the research on hirsutism therapy considers the degree of hirsutism score based on the modified Ferriman-Gallwey score. Despite the fact that the standard hirsutism score is greater than eight, many people with lower scores suffer from the disease. They are looking for cosmetic treatments to get rid of or reduce their extra hair. As a result, pharmacologic treatment is recommended for any woman with known hyperandrogenemia who uses hair removal techniques.

\section{Non-pharmacological methods}

\section{Lifestyle therapies}

In women with polycystic ovarian syndrome, lifestyle interventions are the primary line of therapy, especially if they are overweight [33]. Obese women with the polycystic ovarian syndrome who lose more than $5 \%$ of their original body weight had a substantial improvement in their biochemical profile, including lower testosterone, higher sex hormone-binding globulin, and lower
Ferriman-Gallway scores. Because hair follicles have a half-life of up to six months and lifetime therapy may be required to prevent a recurrence, women should be told not to expect recovery for at least three to six months after therapy begins. Improvement in hirsutism scores can be used to gauge therapy effectiveness, and no additional hormonal testing is necessary.

\section{Epilation}

\section{Physical and chemical epilation}

Most women utilise at-home treatments like as shaving, electric epilating, cold or hot waxes, or chemical epilation. These procedures are inexpensive and simple to apply, but waxing is unpleasant, and all of them are linked to skin irritation and folliculitis. Shaving, on the other hand, is a common misconception that causes hair to grow faster and thicker. To reduce side effects, patients might be taught how to use a clean shaving blade, swab with cotton soaked in a mild boric acid solution, and apply a hydrocortisone lotion to reduce irritation following epilation.

\section{Electro-epilation (Electrolysis)}

A probe is inserted into the hair follicle and destroys it by galvanic electrolysis (direct current) or thermolysis (high-frequency alternating current). The outcomes are very operator-dependent. The regeneration rate is around $40 \%$ [20]. However, it is the most effective treatment for grey hair.

\section{Photo epilation}

Hormonal treatment can slow the progression of the disease, but it only has a minor impact on hair growth reversal. To decrease the quantity of hair, photo epilation or electrolysis is required. The objective of laser or intense pulse light (IPL) treatment for hirsutism is to destroy the hair follicle and lower the hair shaft diameter without harming surrounding tissues, a technique known as selective photothermolysis. When laser light is absorbed by a chromophore, it is converted to heat, causing thermal damage to the target. Melanin is the chromophore for hair removal. Melanin is a pigment that absorbs light in the red and infrared spectra (600-1200 $\mathrm{nm}$ ). In order to determine selective photothermolysis, the pulse width is crucial [34]. To prevent collateral thermal damage, the laser pulse duration must be less than the hair follicle's thermal relaxation period [35]. The time it takes for heated tissue to drop to half of its peak temperature is known as the thermal relaxation period [36]. For terminal hair follicles measuring $200-300 \mathrm{~m}$, the hair follicle thermal relaxation period is $40-100 \mathrm{~ms}$ [37]. The peak temperature within the target structure is determined by the fluence, or energy density $(\mathrm{J} / \mathrm{cm} 2)$. The quantity of heat absorbed is influenced by the frequency and length of the pulses. Both the bulb and the bulge must be addressed in order to kill the hair follicle. Because the goal is melanin, the optimum results are obtained when the skin is light and the hair is dark; moreover, those with darker skin are at a higher risk of thermal blisters and hyperpigmentation. Higher fluences have been linked to more permanent hair removal, as well as higher adverse effects. Treatment fluences recommended for inexperienced operators are adjusted to a safe level, although they are not always successful. Perifollicular erythema and edema may be a better clinical outcome for selecting the best therapy fluence for the individual patient [38]. Combining Eflornithine cream and laser therapy appears to improve treatment outcomes [39].

\section{Mechanical and cosmetic means of treating hirsutism}

Shaving, bleaching, or chemical depilation may be used to reduce undesirable hairs temporarily. Shaving may result in a blunt hair end that feels like stubble, but it does not cause hirsutism to worsen.

Bleaching is beneficial, especially for hair growth that is restricted to a small area. Although helpful, depilating treatments can cause persistent skin irritation and even increase hair growth if used excessively or indiscriminately. In androgenized skin regions, plucking and/or waxing should be avoided since these procedures can cause folliculitis and stress to the hair shaft, resulting in ingrown hairs and additional skin damage.

Electrolytic and laser photothermolysis are two methods for permanently destroying hair follicles that produce undesirable hairs. 
Selective fractional photothermolysis is a technique used in laser hair removal that causes thermal damage to the hair follicle without harming nearby tissues.

Depending on the type of laser or light source used, laser hair removal may be divided into three categories [40]:

1 Red light system (694 nanometer ruby),

2 Infrared light systems (1064 nanometer neodymium: yttriumaluminum-garnet), and

3 Sources of intense pulsed light (590-1200 nanometer).

Patients with Fitzpatrick skin colours I-IV (lighter complexion) and darker pigmented hairs had the best results with laser hair removal. Repeated treatments are required, and total alopecia is unusual. [41]

\section{Pharmacological methods}

\section{Flutamide}

The Food and Drug Administration has authorised this androgen receptor blocker as adjuvant therapy for prostate cancer. In dosages of $500 \mathrm{mg}$ daily, it is an effective therapy for hirsutism. The FG score can be reduced by up to $40 \%$. Greenish urine, severe dryness of skin or scalp hair, liver enzyme abnormalities, and, in rare cases, lethal hepatotoxicity are also side effects [42].

\section{Cyproterone acetate}

Cyproterone acetate is a powerful progestin that lowers circulation testosterone and androstenedione levels via lowering circulating $\mathrm{LH}$ levels. It works to counteract the effects of androgens at the peripheral level, making it an effective therapy for hirsutism. At the treatment of hirsutism, cyproterone acetate in dosages of 50-100 $\mathrm{mg}$ per day g ethinyl estradiol is as effective as spironolactone (100 mg per day) and an OC when coupled with 30-35. In contrast, an OC comprising cyproterone acetate ( $2 \mathrm{mg}$ per day) in g per day) was less effective than $100 \mathrm{mg}$ per day of spironolactone in combination with ethinyl estradiol (35 effective than $100 \mathrm{mg}$ per day of spironolactone, with side effects including adrenal insufficiency and loss of libido[43].

\section{Finasteride}

Finasteride is a 5-reductase inhibitor that has been authorised by the FDA for the treatment of benign prostatic hyperplasia. In dosages of $5 \mathrm{mg}$ per day, it is helpful in treating hirsutism in women, however, it may be less efficient than androgen receptor blockers. The FG score can be reduced by up to $17 \%$. Although teratogenicity (feminization of a male baby) is a serious issue, it has the fewest adverse effects of all the medicines used to treat hirsutism [44].

\section{Oestrogen-progestin oral contraceptives combination}

Oral contraceptives containing oestrogen and progestin decrease hair development in 60-100\% of hyperandrogenic women and are considered first-line treatment. Oral contraceptives reduce hirsutism by inhibiting luteinising hormone secretion and thus luteinising hormone-dependent ovarian androgen production, as well as stimulating sex hormone-binding globulin production by the liver due to the contraceptive's oestrogenic effects, resulting in lower serum concentrations of free testosterone and other sex hormone-binding globulins. Starting therapy with a formulation containing a low dosage of oestrogen and a non-androgenic progestin such desogestrel or norgestimate $[45,46]$ is recommended. An unequivocal contraindication to the oral contraceptive is a history of venous thrombosis [47].

\section{other hormonal therapies}

Glucocorticoid therapy, for example, can reduce adrenal androgen production. These medications are used to treat hirsutism caused by all types of congenital and late-onset adrenal hyperplasia [48]. Administration of a gonadotropin-releasing hormone agonist, such as leuprolide, suppresses gonadotropin and hence ovarian androgen production in women with hirsutism, resulting in hair growth slowing but also oestrogen insufficiency. The use of a gonadotropin-releasing hormone agonist in conjunction with either low-dose oestrogen and progesterone or an oral contraceptive removes the negative effects of oestrogen shortage while maintaining the gonadotropin-releasing hormone agonist's advantages. An agonist plus an oral contraceptive, on the other hand, is less effective than flutamide or cyproterone plus an oral contraceptive. Metformin and rosiglitazone improved insulin sensitivity in both obese and lean polycystic ovarian syndrome patients (BMI $25 \mathrm{~kg} / \mathrm{m} 2$ ). These medications may also lower testosterone levels and restore normal menstrual cyclicity [49]

\section{Anti-androgens}

Anti-androgens are an efficient hirsutism therapy. In women with severe hirsutism, combined therapy with an oral contraceptive and an anti-androgen is more likely to be successful than either treatment alone. Spironolactone prevents testosterone from attaching to its receptors, preventing it from acting. It also reduces ovarian production and testosterone clearance. Cyproterone acetate is an anti-androgenic progestin that can be used alone or in combination with an oral contraceptive to treat hirsutism. To prevent bone loss, cyproterone acetate is probably best taken in conjunction with oestrogen. This medication is not licenced in the United States, although it is accessible in Europe and Canada as a combination oral contraceptive (Diane) that contains ethinyloestradiol and cyproterone acetate. A dosage of $2 \mathrm{mg}$ administered in conjunction with $21 \mathrm{~d}$ of ethinyloestradiol $35 \mathrm{l}$ gram(Diane) has been proven to alleviate symptoms in over $50 \%$ of instances of mild-to-moderate hirsutism. Cyproterone acetate is administered at a dosage of 25-100 mg in combination with Diane or $30 \mathrm{l}$ gram ethinyl oestradiol for a 21-day cycle in more severe hirsutism [50].

\section{Agonist of GnRH}

A long-acting $\mathrm{GnRH}$ protagonist can effectively suppress ovarian hormone production. When the effects of oestrogen and progestin constitute the source of the disease, such as in the treatment of endometriosis or leiomyomata, this therapy is very beneficial. With hirsutism, however, this is not the case. In reality, oestrogen has a positive influence on hirsutism treatment. GnRH protagonist are rarely used alone to treat ovarian androgen-related hirsutism because of this. They can be beneficial in determining whether androgen secretion is dependent on gonadotropins or not [51].

\section{Eflornithine hydrochloride}

Eflornithine is an orithine decarboxylase inhibitor that is irreversible. It catalyses the rate-limiting step in the production of follicular polyamines, which is required for hair development. It does not eliminate hair, but it does slow down its development. Many nations have authorised a topical Eflornithine hydrochloride cream (13.9 percent) for the treatment of unsightly facial hair in women. Skin irritation has only been documented with overuse under experimental circumstances, and systemic absorption is minimal. Itching and dry skin have been reported as side effects in clinical trials [20]. The cream's merits include its capacity to prevent hair growth of any hue, but its drawbacks include the need for twicedaily applications and ongoing usage to maintain effects [14].

\section{Insulin sensitizers}

In many women with PCOS, treatment of insulin resistance, typically by weight loss or the use of metformin or thiazolidinediones, has been shown to improve hyperandrogenemia and ovulatory function. Its efficacy of the treatment of PCOS-related hirsutism, on the other hand, is less apparent. Insulin-sensitizing medications may help with PCOS-related hirsutism in a small way [52].

\section{Spironolactone}

Spironolactone is a mild diuretic and an aldosterone antagonist. It also competes with androgens for the-reductase and the androgen receptor that binds to sex hormones, globulin. It also has a suppressive impact on a number of enzymes involved in androgen production. Spironolactone, regardless of the degree of hyperandrogenemia, is a very effective treatment for hirsutism. With $100 \mathrm{mg}$ of spironolactone for $6 \mathrm{mo}$, the FG score is reduced by $19 \%$. Although daily dosages of $100 \mathrm{mg}$ are typically beneficial for the 
treatment of hirsutism, in extremely hirsute or obese women, larger doses (200-300 mg per day) may be preferable [53].

\section{Follow-UP}

Although clinical response will be the major measure tracked, circulating androgen and sex hormone-binding globulin levels may be evaluated during treatment to determine the appropriateness of hormonal therapy. It's crucial to note that hirsutism may improve with therapy only after 6 or 8 mo of treatment. Furthermore, patients should be informed that the primary goal of hormone therapy is to address the underlying issue, inhibit new hair development, and maybe decrease the growth of existing terminal hairs. Hormonal treatment alone may cause terminal hair thinning and loss of pigmentation, but it will not reverse the terminalization of baby hairs that have already been converted [54]

Electrolysis or laser hair removal will be required to completely remove the already androgenized hair follicles. After hormone treatment has had a chance to suppress hair growth, generally, after 6-12 mo, it is better to begin more definitive hair destruction and removal. Because the menopausal ovary still generates a significant quantity of androgen and the antagonistic effects of circulating estrogens are decreased, some individuals' hirsutism may develop after menopause. This is especially true if individuals are not taking hormone replacement treatment orally. Patients' hair growth may improve as they become older, due to the widespread loss of hair follicles and the decrease in androgens that comes with age [54].

Overall, despite the fact that hirsutism is a common and painful condition that frequently indicates an underlying endocrine problem, most patients will benefit from a methodical approach to assessment and the use of combination medication.

\section{FUNDING}

Nil

\section{AUTHORS CONTRIBUTIONS}

All the authors have contributed equally.

\section{CONFLICTS OF INTERESTS}

Declared none

\section{REFERENCES}

1. Wendelin DS, Pope DN, Mallory SB. Hypertrichosis. J Am Acad Dermatol. 2003;48(2):161-79; quiz $180 . \quad$ doi: 10.1067/mjd.2003.100, PMID 12582385.

2. Bode D, Seehusen DA, Baird D. Hirsutism in women. Am Fam Physician. 2012;85(4):373-80. PMID 22335316.

3. Vulink AJ, Ten Bokkel Huinink D. Acquired hypertrichosis lanuginosa: a rare cutaneous paraneoplastic syndrome. J Clin Oncol. 2007;25(12):1625-6. doi: 10.1200/JC0.2007.10.6963, PMID 17443005.

4. Ferriman D, Gallwey JD. Clinical assessment of body hair growth in women. J Clin Endocrinol Metab. 1961;21:1440-7. doi: 10.1210/jcem-21-11-1440, PMID 13892577.

5. Azziz R, Carmina E, Sawaya ME. Idiopathic hirsutism. Endocr Rev. 2000;21(4):347-62. doi: 10.1210/edrv.21.4.0401, PMID 10950156.

6. Yildiz BO. Diagnosis of hyperandrogenism: clinical criteria. Best Pract Res Clin Endocrinol Metab. 2006;20(2):167-76. doi: 10.1016/j.beem.2006.02.004, PMID 16772149.

7. Glintborg D, Andersen M. An update on the pathogenesis, inflammation, and metabolism in hirsutism and polycystic ovary syndrome. Gynecol Endocrinol. 2010;26(4):281-96. doi: 10.3109/09513590903247873. PMID 20141388.

8. PCOS and hyperandrogenism from the Textbook of Williams Gynecology. 1st ed. 2021. p. 383-99.

9. Pritts EA. Treatment of the infertile patient with polycystic ovarian syndrome. Obstet Gynecol Surv. Sep 2002;57(9):587-97. doi: 10.1097/00006254-200209000-00022, PMID 12218667.

10. Dunaif A. Hyperandrogenic anovulation PCOS: a unique disorder of insulin action associated with an increased risk of non-insulin-dependent diabetes mellitus. Am J Med. Jan 16 1995;98:(IA335-39).

11. Conway GS, Agrawal R, Betteridge DJ, Jacobs HS. Risk factors for coronary artery disease in lean and obese women with the polycystic ovary syndrome. Clin Endocrinol (Oxf). 1992;37(2):119-25. doi: 10.1111/j.1365-2265.1992.tb02295.x, PMID 1395062.

12. Hunter MH, Carek PJ. Evaluation and treatment of women with hirsutism. Am Fam Physician. 2003 Jun 15;67(12):2565-72. PMID 12825846.

13. Gheorghisan-Galateanu A, Fica S, Terzea DC, Caragheorgheopol A, Horhoianu V. Sertoli-Leydig cell tumor- a rare androgen secreting ovarian tumor in postmenopausal women. Case report and review of literature. J Cell Mol Med. Oct 2003;4:461-71. doi: 10.1111/j.1582-4934.2003.tb00249.x, PMID 14754515.

14. Brodell LA, Mercurio MG. Hirsutism: diagnosis and management. Gend Med. 2010;7(2):79-87. doi: 10.1016/j.genm.2010.04.002, PMID 20435271.

15. Martin KA, Chang RJ, Ehrmann DA, Ibanez L, Lobo RA, Rosenfield RL, Shapiro J, Montori VM, Swiglo BA. Evaluation and treatment of hirsutism in premenopausal women: an endocrine society clinical practice guideline. J Clin Endocrinol Metab. 2008;93(4):1105-20. doi: 10.1210/jc.2007-2437, PMID 18252793.

16. Somani N, Harrison S, Bergfeld WF. The clinical evaluation of hirsutism. Dermatol Ther. 2008;21(5):376-91. doi: 10.1111/j.1529-8019.2008.00219.x, PMID 18844715.

17. Franks S, McCarthy M. Genetics of ovarian disorders: polycystic ovary syndrome. Rev Endocr Metab Disord. 2004;5(1):69-76 doi: 10.1023/B:REMD.0000016125.05878.96, PMID 14966390.

18. Goodman NF, Cobin RH, Futterweit W, Glueck JS, Legro RS, Carmina E. American Association of Clinical Endocrinologists (AACE), American College of Endocrinology (ACE), Androgen Excess and PCOS Society (AES). American Association of clinical endocrinologists, American College of endocrinology, and androgen excess and PCOS society disease state clinical review: guide to the best practices in the evaluation and treatment of polycystic ovary syndrome- part 1. Endocr Pract. 2015;21(11):1291-300. doi: 10.4158/EP15748.DSC, PMID 26509855.

19. Blume Peytavi U. How to diagnose and treat medically women with excessive hair. Dermatol Clin. 2013;31(1):57-65. doi: 10.1016/j.det.2012.08.009, PMID 23159176.

20. Dokras A. Mood and anxiety disorders in women with PCOS. Steroids. 2012;77(4):338-41. 10.1016/j.steroids.2011.12.008, PMID 22178257.

21. Krępuła K, Bidzinska Speichert B, Lenarcik A, Tworowska Bardzinska U. Psychiatric disorders related to polycystic ovary syndrome. Endokrynol Pol. 2012;63(6):488-91. PMID 23339008.

22. Goodman NF, Bledsoe MB, Cobin RH, Futterweit W, Goldzieher JW, Petak SM, Smith KD, Steinberger E. American association of clinical endocrinologists hyperandrogenic disorders task force. American Association of Clinical Endocrinologists medical guidelines for the clinical practice for the diagnosis and treatment of hyperandrogenic disorders. [American Association of Clinical Endocrinologists medical guidelines for clinical practice for the diagnosis and treatment of hyperandrogenic disorders]. Endocr Pract. 2001;7(2):120-34. doi: 10.4158/EP.7.2.120, PMID 12940239.

23. Polson DW, Adams J, Wadsworth J, Franks S. Polycystic ovaries- A common finding in normal women. Lancet. 1988;1(8590):870-72. doi: 10.1016/s0140-6736(88)91612-1, PMID 2895373.

24. Legro RS, Driscoll D, Strauss JF III, Fox J, Dunaif A. Evidence for a genetic basis for hyperandrogenemia in polycystic ovary syndrome. Proc Natl Acad Sci USA. 1998;95(25):14956-60. doi: 10.1073/pnas.95.25.14956, PMID 9843997.

25. Lin-Su K, Nimkarn S, New MI. Congenital adrenal hyperplasia in adolescents: diagnosis and management. Ann NY Acad Sci. 2008;1135:95-8. doi: 10.1196/annals.1429.021, PMID 18574213.

26. Reingold SB, Rosenfield RL. The relationship of mild hirsutism or acne in women to androgens. Arch Dermatol. 1987;123(2):209-12, PMID 2949707. 
27. Azziz R, Sanchez LA, Knochenhauer ES, Moran C, Lazenby J, Stephens KC, Taylor K, Boots LR. Androgen excess in women: experience with over 1000 consecutive patients. J Clin Endocrinol Metab. 2004;89(2):453-62. doi: 10.1210/jc.2003031122 , PMID 14764747.

28. Wild RA, Umstot ES, Andersen RN, Ranney GB, Givens JR. Androgen parameters and their correlation with body weight in one hundred thirty-eight women thought to have hyperandrogenism. Am J Obstet Gynecol. 1983;146(6):602-6. doi: 10.1016/0002-9378(83)90998-5, PMID 6223527.

29. Rosenfield RL. Hirsutism and the variable response of the pilosebaceous unit to androgen. J Investig Dermatol Symp Proc. 2005;10(3):205-8. doi: 10.1111/j.1087-0024.2005.10106.x, PMID 16382665.

30. Marynick SP, Chakmakjian ZH, McCaffree DL, Herndon JH Jr. Androgen excess in cystic acne. N Engl J Med. 1983;308(17):981-6. doi: 10.1056/NEJM198304283081701, PMID 6220224.

31. Azziz R, Dewailly D, Owerbach D. Clinical review 56: Nonclassic adrenal hyperplasia: current concepts. J Clin Endocrinol Metab. 1994;78(4):810-5. doi: 10.1210/jcem.78.4.8157702, PMID 8157702.

32. Chang RJ, Katz SE. Diagnosis of polycystic ovary syndrome. Endocrinol Metab Clin North Am. 1999;28(2):397-408, vii. doi: 10.1016/s0889-8529(05)70076-1, PMID 10352925.

33. Schmidt JB, Lindmaier A, Spona J. Hyperprolactinemia and hypophyseal hypothyroidism as cofactors in hirsutism and androgen-induced alopecia in women. Hautarzt. 1991;42(3):168-72. PMID 1905280.

34. Ibanez L, de Zegher F. Ethinylestradiol-drospirenone, flutamide-metformin, or both for adolescents and women with hyperinsulinemic hyperandrogenism: opposite effects on adipocytokines and body adiposity. J Clin Endocrinol Metab. 2004;89(4):1592-7. doi: 10.1210/jc.2003-031281, PMID 15070917.

35. Anderson RR, Parrish JA. Selective photothermolysis: precise microsurgery by selective absorption of pulsed radiation. Science. 1983;220(4596):524-7. doi: 10.1126/science.6836297, PMID 6836297.

36. Sanchez LA, Perez M, Azziz R. Laser hair reduction in the hirsute patient: a critical assessment. Hum Reprod Update. 2002;8(2):169-81. doi: 10.1093/humupd/8.2.169, PMID 12099632.

37. Gan SD, Graber EM. Laser hair removal: a review. Dermatol Surg. 2013;39(6):823-38. doi: 10.1111/dsu.12116, PMID 23332016.

38. Hamzavi I, Tan E, Shapiro J, Lui H. A randomized bilatera vehicle-controlled study of eflornithine cream combined with laser treatment versus laser treatment alone for facial hirsutism in women. J Am Acad Dermatol. 2007;57(1):54-9. doi: 10.1016/j.jaad.2006.09.025, PMID 17270315.

39. Ibrahimi OA, Avram MM, William HCHanke CW, Suzanne L Kilmer SL, Anderson RR, Rox Anderson R. Laser hair removal. Dermatol Ther. 2011;24(1):94-107. doi: 10.1111/j.15298019.2010.01382.x, PMID 21276162.

40. Nanni CA, Alster TS. Laser-assisted hair removal: side effects Qswitched Nd: YAG, long-pulsed ruby, and alexandrite lasers. J Am Acad Dermatol. 1999;41(2 Pt 1):165-71. doi: 10.1016/s0190-9622(99)70043-5, PMID 10426883.
41. Cusan L, Dupont A, Gomez JL, Tremblay RR, Labrie F. Comparison of flutamide and spironolactone in the treatment of hirsutism: a randomized controlled trial. Fertil Steril. 1994;61(2):281-7. doi: 10.1016/s0015-0282(16)56518-2, PMID 8299783.

42. Van der Spuy ZM, Le Roux PA. Cyproterone acetate for hirsutism. Cochrane Database Syst Rev. 2003;(4):CD001125. doi: 10.1002/14651858.CD001125, PMID 14583927.

43. Wong IL, Morris RS, Chang L, Spahn MA, Stanczyk FZ, Lobo RA A prospective randomized trial comparing finasteride to spironolactone in the treatment of hirsute women. J Clin Endocrinol Metab. 1995;80(1):233-8. doi: 10.1210/jcem.80.1.7829618, PMID 7829618.

44. Burkman RT Jr. The role of oral contraceptives in the treatment of hyperandrogenic disorders. Am J Med. 1995;98(1A):130S6130S-6S. doi: 10.1016/s0002-9343(99)80071-0, PMID 7825633.

45. Mastorakos G, Koliopoulos C, Creatsas G. Androgen and lipid profiles in adolescents with polycystic ovary syndrome who were treated with two forms of combined oral contraceptives. Fertil Steril. 2002;77(5):919-27. doi: 10.1016/s00150282(02)02993-x, PMID 12009344.

46. Olaah KS. The modern management of hirsutism. Rev Gynaecol Pract. 2004;4(4):211-20. doi: 10.1016/j.rigp.2004.05.001.

47. Yilmaz M, Biri A, Karakocç A, Syeyedahmad Seyedalinaghi Toruner F, Bingol B, Cakir N, Tiras B, Ayvaz G, Arslan M. The effects of rosiglitazone and metformin on insulin resistance and serum androgen levels in obese and lean patients with polycystic ovary syndrome. J Endocrinol Invest. 2005;28(11):1003-8. doi: 10.1007/BF03345339, PMID 16483179.

48. Cumming DC, Yang JC, Rebar RW, Yen SS. Treatment of hirsutism with spironolactone. JAMA. 1982;247(9):1295-8, PMID 7199587

49. Sahin Y, Dilber S, Kelesștimur F. Comparison of diane 35 and diane 35 plus finasteride in the treatment of hirsutism. Fertil Steril. 2001;75(3):496-500. doi: 10.1016/s00150282(00)01764-7, PMID 11239530.

50. Nikolaou D, Gilling Smith C. Hirsutism. Curr Obstet Gynaecol. 2005;15(3):174-82. doi: 10.1016/j.curobgyn.2005.03.006.

51. Loriaux DL. An approach to the patient with hirsutism. J Clin Endocrinol Metab. 2012;97(9):2957-68. doi: 10.1210/jc.20112744, PMID 22962669.

52. Cosma M, Swiglo BA, Flynn DN, Kurtz DM, Labella ML, Mullan RJ, Elamin MB, Erwin PJ, Montori VM. Clinical review: Iinsulin sensitizers for the treatment of hirsutism: A systematic review and metaanalyses of randomized controlled trials. J Clin Endocrinol Metab. 2008;93(4):1135-42. doi: 10.1210/jc.20072429, PMID 18252787

53. Helfer EL, Miller JL, Rose LI. Side-effects of spironolactone therapy in the hirsute woman. J Clin Endocrinol Metab. 1988;66(1):208-11. doi: 10.1210/jcem-66-1-208, PMID 3335604.

54. Winters SJ, Talbott E, Guzick DS, Zborowski J, McHugh KP. Serum testosterone levels decrease in middle age in women with the polycystic ovary syndrome. Fertil Steril. 2000;73(4):724-9. doi: 10.1016/s0015-0282(99)00641-x, PMID 10731532. 